\title{
Trypsin-specific inhibitors from the basidiomycete Clitocybe nebularis with regulatory and defensive functions
}

\begin{abstract}
Correspondence
Jerica Sabotič

Jerica.Sabotic@ijs.si
\end{abstract}

Received 16 July 2009

Revised 17 August 2009

Accepted 18 August 2009

\author{
Petra Avanzo, ${ }^{1}$ Jerica Sabotič, ${ }^{1}$ Sabina Anžlovar, ${ }^{1} \dagger$ Tatjana Popovič, ${ }^{1}$ \\ Adrijana Leonardi, ${ }^{2}$ Roger H. Pain, ${ }^{1}$ Janko Kos ${ }^{1,3}$ and Jože Brzin ${ }^{1}$ \\ ${ }^{1}$ Department of Biotechnology, Jožef Stefan Institute, Jamova 39, SI-1000 Ljubljana, Slovenia \\ ${ }^{2}$ Department of Molecular and Biomedical Sciences, Jožef Stefan Institute, Jamova 39, SI-1000 \\ Ljubljana, Slovenia \\ ${ }^{3}$ Faculty of Pharmacy, University of Ljubljana, Aškerčeva 7, SI-1000 Ljubljana, Slovenia
}

\begin{abstract}
We have isolated serine protease inhibitors from the basidiomycete Clitocybe nebularis, CnSPIs, using trypsin affinity chromatography. Full-length gene and cDNA sequences were determined for one of them, named cnispin, and the recombinant protein was expressed in Escherichia coli at high yield. The primary structure and biochemical properties of cnispin are very similar to those of the Lentinus edodes serine protease inhibitor, until now the only member of the 166 family of protease inhibitors in the MEROPS classification. Cnispin is highly specific towards trypsin, with $K_{\mathrm{i}}$ in the nanomolar range. It also exhibited weaker inhibition of chymotrypsin and very weak inhibition of subtilisin and kallikrein; other proteases were not inhibited. Inhibitory activity against endogenous proteases from $C$. nebularis revealed a possible regulatory role for $\mathrm{CnSPIs}$ in the endogenous proteolytic system. Another possible biological function in defence against predatory insects was indicated by the deleterious effect of CnSPIs on the development of larvae of Drosophila melanogaster. These findings, together with the biochemical and genetic characterization of cnispin, suggest a dual physiological role for this serine protease inhibitor of the 166 MEROPS family.
\end{abstract}

\section{INTRODUCTION}

Serine proteases are found in all kingdoms of life, including viral genomes. They constitute more than one-third of all known proteolytic enzymes and, according to the MEROPS classification, are grouped into 13 clans, based on catalytic mechanism, and 40 families, based on common ancestry. Proteases of clan PA are widely represented in eukaryotes, with the exception of plants and fungi, in which other clans (mainly SB and SC) have more representatives (Page \& Di Cera, 2008; Rawlings et al., 2008). Serine proteases are also the predominant proteolytic enzymes in basidiomycete

tPresent address: Department of Biology, Biotechnical Faculty, University of Ljubljana, Večna pot 111, Sl-1000 Ljubljana, Slovenia.

Abbreviations: B, N-benzoyl; BAPNA, N-benzoyl-DL-arginine- $p$-nitroanilide; BOC, butoxycarbonyl; $\mathrm{CD}$, circular dichroism; CnSPI, Clitocybe nebularis serine protease inhibitor; (r)Cnp, (recombinant) cnispin; LeSPI, Lentinus edodes serine protease inhibitor; MCA, 7-(4-methyl)coumarylamide; PAA, polyacrylamide; pNA, $p$-nitroanilide; UTR, untranslated region; Z, benzyloxycarbonyl.

The GenBank/EMBL/DDBJ accession numbers for the sequences reported in this paper are FJ478178 and GQ141891.

Supplementary data are available with the online version of this paper. mushrooms. An even larger proportion of gelatinolytic activities could not be classified, thus representing potentially unique proteases with unknown types of catalytic mechanism, demonstrating mushrooms as an important source of different types of proteolytic enzymes (Sabotič et al., 2007b).

Serine proteases are involved in various physiological and pathophysiological processes, including digestion, blood coagulation, fibrinolysis, development, fertilization, apoptosis, immunity and cancer progression, playing key roles in human health and disease (Nyberg et al., 2006; Page \& Di Cera, 2008). The serine proteases trypsin and chymotrypsin are responsible for the initial digestion of ingested proteins in humans (Whitcomb \& Lowe, 2007) and in invertebrates (Muhlia-Almazan et al., 2008). Similarly, serine proteases play very important nutritional roles in higher fungi. In addition to their obvious proteolytic role during growth in nitrogen-limiting conditions, serine proteases from Pleurotus ostreatus act as regulators of other extracellular proteases, and of laccases, which are involved in the degradation of lignin (Faraco et al., 2005; Palmieri et al., 2001). In Agaricus bisporus, serine proteases regulate activation of tyrosinases, enzymes that catalyse 
oxidation of phenols (Espin et al., 1999). A serine protease from A. bisporus is involved in resource recycling during senescence in fruiting bodies, mobilizing nutrients from the stipe to reproductive spore-bearing tissues in the cap (Burton et al., 1997). The nutritional role of this enzyme was confirmed by following the regulation of its expression in response to available nitrogen in A. bisporus and Coprinopsis cinerea. A role in fruiting body development was also suggested for serine proteases in both mushrooms (Heneghan et al., 2009). Serine proteases are also important pathogenicity factors. For the mushroom pathogen Verticillium fungicola, subtilisin-type serine proteases are important for the attack of hyphal cell walls (St Leger et al., 1997). Furthermore, dipteran larvae are major mushroom insect pests, and have serine proteases as the predominant digestive proteolytic enzymes (Coles et al., 2002; Terra \& Ferreira, 1994).

Specific protease inhibitors are essential in regulating endogenous proteolytic processes and protecting against exogenously introduced proteases of pathogens (Vandeputte-Rutten \& Gros, 2002). Inhibitory activity against trypsin has been detected in fruiting bodies of basidiomycete fungi (Gzogian et al., 2005; Vetter, 2000), yet only a few serine protease inhibitors from mushrooms have been isolated and characterized. These include two isomeric inhibitors of serine proteases IA-1 and IA-2 from P. ostreatus (Dohmae et al., 1995) belonging to family I9 in the MEROPS classification, a serine proteinase inhibitor from Lentinus edodes (Odani et al., 1999) that inhibits trypsin and chymotrypsin, belonging to family I66, and proteinase $\mathrm{K}$ inhibitor from Trametes versicolor (Zuchowski \& Grzywnowicz, 2006), not assigned to a MEROPS inhibitor family.

We have previously isolated and characterized a novel family of inhibitors of cysteine proteases from the basidiomycetes Clitocybe nebularis (Brzin et al., 2000; Sabotič et al., 2006, 2007a) and Macrolepiota procera (Sabotič et al., 2009). Here we describe biochemical properties and evidence for the biological function of new serine protease inhibitors, CnSPIs (Clitocybe nebularis serine protease inhibitors), isolated from C. nebularis, and genetic and biochemical characterization of one of these inhibitors, cnispin (Cnp), which has been heterologously expressed in Escherichia coli.

\section{METHODS}

Enzymes and substrates. Bovine trypsin (EC 3.4.21.4), chymotrypsin (EC 3.4.21.1), porcine kallikrein (EC 3.4.21.35) and porcine pepsin (EC 3.4.23.1) were from Sigma, bovine thrombin (EC 3.4.21.5) from Calbiochem, Bacillus subtilis subtilisin (EC 3.4.21.62) from Boehringer Mannheim and porcine elastase (EC 3.4.21.36) from Serva. $2 \times$ crystallized papain (EC 3.4.22.2) from Sigma was further purified by affinity chromatography (Blumberg et al., 1970). Z-PheArg-MCA [7-(4-methyl)-coumarylamide], Suc-Ala-Ala-Pro-PheMCA, Boc-Val-Pro-Arg-MCA, H-Pro-Phe-Arg-MCA, Suc-Ala-AlaAla-MCA and B-Arg-pNA ( $N$-benzoyl-DL-arginine-p-nitroanilide, BAPNA) were from Bachem.
Fungal material. Fruiting bodies of $C$. nebularis were collected from their natural habitat in Kras forest (Slovenia) and frozen at $-20{ }^{\circ} \mathrm{C}$ until use. Tissue from different parts of the basidiocarp and mycelium was prepared as described previously (Sabotič et al., 2006). Mycelium was grown in $1.5 \%(\mathrm{w} / \mathrm{v})$ malt extract medium in the dark at $30{ }^{\circ} \mathrm{C}$. The mycelium is kept in the collection of fungi, lichens and higher plants at the Slovenian Forestry Institute, Ljubljana, Slovenia.

Isolation of serine protease inhibitors. Frozen fruiting bodies or basidiocarps of C. nebularis were homogenized in buffer A $(0.02 \mathrm{M}$ Tris/HCl, pH 7.5, $0.3 \mathrm{M} \mathrm{NaCl}$ ) containing $4 \mathrm{mM} \mathrm{Na}_{2} \mathrm{~S}_{2} \mathrm{O}_{3}$ (reducing agent). The homogenate was filtered and centrifuged for $15 \mathrm{~min}$ at $3400 \mathrm{~g}$ at $4{ }^{\circ} \mathrm{C}$. The supernatant was applied to a trypsin affinity chromatography column $(3.5 \times 15 \mathrm{~cm})$ prepared according to the manufacturer's instructions (Pharmacia) and equilibrated with buffer A. Bound inhibitory fractions were eluted with $0.01 \mathrm{M} \mathrm{HCl}$, pooled, neutralized with $2 \mathrm{M}$ Tris/ $\mathrm{HCl}$ and concentrated by ultrafiltration (UM-10, Amicon). Serine protease inhibitors were additionally purified on a Sephacryl S-200 (Amersham Pharmacia Biotech) column $(4 \times 110 \mathrm{~cm})$ in buffer A at a flow rate of $52.8 \mathrm{ml} \mathrm{h}^{-1}$.

Analysis of endogenous serine proteases. Crude protein extract of C. nebularis basidiocarps was prepared by extraction with $0.5 \mathrm{M}$ sodium acetate buffer, $\mathrm{pH} 4.5$, containing $0.3 \mathrm{M} \mathrm{NaCl}, 50 \mathrm{mM}$ EDTA and $0.05 \%(\mathrm{w} / \mathrm{w}) \mathrm{Na}_{2} \mathrm{~S}_{2} \mathrm{O}_{3}$. Insoluble material was removed by centrifugation at $9000 \mathrm{~g}$ for $15 \mathrm{~min}$. The resulting supernatant was concentrated by ultrafiltration (UM-3, Amicon) and applied to a Sepharose S-200 size-exclusion chromatography column $(1.5 \times 130 \mathrm{~cm})$ equilibrated with $0.1 \mathrm{M}$ sodium acetate buffer, $\mathrm{pH} 5.5$, containing $0.3 \mathrm{M} \mathrm{NaCl}$ and $10 \mathrm{mM}$ EDTA, and eluted at a flow rate of $9.6 \mathrm{ml} \mathrm{h}^{-1}$. Proteolytic activity in fractions was measured using Z-Phe-Arg-MCA as substrate in $0.1 \mathrm{M}$ Tris/HCl, $\mathrm{pH}$ 9, and the influence of different inhibitors was tested. The following inhibitors were used at the indicated final concentration: $4 \mathrm{mM}$ Pefabloc SC [AEBSF; 4-(2-aminoethyl)benzenesulfonyl fluoride hydrochloride, Roche], $100 \mu \mathrm{M}$ chymostatin (Sigma), $30 \mu \mathrm{M}$ E-64 ([(2S,3S)-3carboxyoxirane-2-carbonyl]-L-leucine (4-guanidinobutyl)amide, Peptide Institute) and CnSPIs at $0.02 \mathrm{mg} \mathrm{ml}^{-1}$ and $0.2 \mathrm{mg} \mathrm{ml}^{-1}$.

Insecticidal effect of CnSPIs on Drosophila melanogaster. Insecticidal effect was determined in feeding bioassays as relative mortality of larvae. Protease inhibitors were added to the rearing medium $[3.2 \%(\mathrm{w} / \mathrm{v})$ sucrose, $8.5 \%(\mathrm{w} / \mathrm{v})$ yeast extract, $5 \%(\mathrm{w} / \mathrm{v})$ cornflour, $1.2 \%(\mathrm{w} / \mathrm{v})$ agar, $1.1 \%(\mathrm{v} / \mathrm{v})$ p-methylhydroxybenzoic acid, $0.5 \%(\mathrm{v} / \mathrm{v})$ propionic acid] before pouring into $1 \times 5 \mathrm{~cm}$ tubes. Ten eggs of wild-type D. melanogaster strain Canton S (Indiana University, USA) were deposited on the medium and the tubes were maintained at $25{ }^{\circ} \mathrm{C}$ for 14 days to allow development of larvae into pupae, when their numbers were recorded. Each test was performed in five replicates. Rearing medium containing $0.5 \mathrm{mg} \mathrm{BSA} \mathrm{ml}^{-1}$ was used as a reference assay. The following protease inhibitors were tested at a final concentration of $0.5 \mathrm{mg} \mathrm{ml}^{-1}$ : C. nebularis serine protease inhibitors (CnSPIs), serine protease inhibitor from pumpkin, Cucurbita maxima (CMTI) (Krishnamoorthi et al., 1990), potato serine protease inhibitor (PSPI) (Brzin et al., 1995), potato protease inhibitor 2 (PI2) (Pearce et al., 1982), and C. nebularis cysteine protease inhibitor (clitocypin) (Brzin et al., 2000). Relative mortality (RM) of larvae was determined as $\mathrm{RM}=1-[$ (test mortality)/(reference mortality)] and standard deviation was calculated.

SDS-PAGE and IEF analyses. Proteins were analysed on $12 \%$ $(\mathrm{w} / \mathrm{v})$ polyacrylamide (PAA) gels in denaturing and non-reducing or reducing conditions and visualized using Coomassie brilliant blue staining. Low-molecular-mass markers $14.4-97 \mathrm{kDa}$ (Amersham Pharmacia Biotech) were used for molecular mass estimation. Isoelectric focusing was carried out with a Pharmacia PhastSystem, 
using commercial precast $\mathrm{pH}$ 3-9 gradient gels as described previously (Sabotic et al., 2007a).

Protein sequencing. For the $\mathrm{N}$-terminal sequence analysis, proteins separated by SDS-PAGE were electro-transferred to a PVDF membrane (Millipore), and visualized by Coomassie staining. Bands were excised and sequencing was performed on a Procise 492A Automated Sequencing System (Applied Biosystems).

For internal protein cleavage, the PAA gel was first negatively stained with Zn/imidazole (Ortiz et al., 1992). The band of interest was excised, equilibrated in non-reducing sample buffer for $5 \mathrm{~min}$ and applied onto another PAA gel. SDS-PAGE was stopped when the protein entered the stacking gel. Immediately, $1 \mu \mathrm{g}$ of Staphylococcus aureus V8 protease (EC 3.4.21.19, Miles) was applied and SDS-PAGE was run until proteins reached the boundary between stacking and resolving gel. The cleavage was left to proceed for $1 \mathrm{~h}$ at room temperature. Then SDS-PAGE was run to the end, after which the proteins were electro-blotted and the $\mathrm{N}$-terminal sequences of the resulting fragments determined as described above.

HPLC analysis. The proteins were separated on an HPLC system using an Aquapore BU-300 column (PE Brownlee). The column was washed with $0.1 \%(\mathrm{v} / \mathrm{v})$ trifluoroacetic acid (TFA) in water and the proteins were eluted from the column with a linear gradient of acetonitrile in $0.1 \%$ TFA $(0-90 \%$ in $30 \mathrm{~min})$ at a flow rate of $1 \mathrm{ml}$ $\min ^{-1}$.

Electrospray-ionization mass spectrometry. The molecular masses of the HPLC-purified proteins were determined by electrospray ionization (ESI) mass spectrometry as described previously (Pohleven et al., 2009) on a Q-Tof Premier (Micromass MS Technologies/Waters) mass spectrometer.

Molecular cloning of the gene and cDNA encoding cnispin. Molecular cloning of the cnispin gene ( $c n p)$ and cDNA sequences followed the same steps as those for the C. nebularis lectin (Pohleven et al., 2009). High-molecular-mass genomic DNA was isolated from frozen C. nebularis fruiting bodies as described by Moller et al. (1992), and cDNA synthesis was performed using total RNA, as described previously (Pohleven et al., 2009). Partial cnp gene and cDNA sequences were amplified with forward (CnSPI-D-f) and reverse (CnSPI-D-r) degenerate primers that were constructed using the CADEPCO program (http://sabina.anzlovar.com/calc/cadepco.cgi) (Supplementary Table S1). On the basis of the resulting partial nucleic acid sequences, specific primers (Supplementary Table S1) were designed to amplify the complete gene and cDNA sequences.

To amplify the $3^{\prime}$ end of the cDNA sequence, $3^{\prime}$ rapid amplification of cDNA ends (3' RACE) was carried out using the 3' RACE adaptor primer (Supplementary Table S1) paired with a forward cnp-specific primer CnSPI-RACE-N1 (Supplementary Table S1) in the primary PCR. This was followed by secondary PCR using cnp-specific nested primer CnSPI-RACE-N2 (Supplementary Table S1). The complete cnp gene, with its promoter and terminator regions, was amplified using genome walking libraries as templates, constructed using the GenomeWalker Universal kit (BD Biosciences Clontech) as described previously (Pohleven et al., 2009). In genome walking PCR amplifications, Advantage 2 Polymerase Mix (Clontech) was used with the PCR conditions suggested by the manufacturer. Adaptor primer AP1 and a nested primer AP2, provided by the manufacturer, were paired with nested forward gene-specific primers (CnSPI-term1 and CnSPI-term2; Supplementary Table S1) for downstream amplification, or nested reverse gene-specific primers (CnSPI-prom1 and CnSPI-prom2; Supplementary Table S1) for upstream amplification. Complete $c n p$ gene and cDNA sequences were obtained using primers annealing to the $5^{\prime}$ untranslated region (5' UTR) (CnSPI-f) and 3' UTR (CnSPI-r).
All PCR products were cloned into pGEM-T Easy Vector System I (Promega) and sequenced using the Automated DNA Sequencing Service at Eurofins MWG Operon (Ebersberg, Germany).

Sequence analysis. Sequence analyses and multiple sequence alignments were performed in the BioEdit Sequence Alignment Editor (http://www.mbio.ncsu.edu/bioedit/bioedit.html). Promoter analysis was performed using TESS (Transcription Element Search System) at http://www.cbil.upenn.edu/cgi-bin/tess/tess. The deduced amino acid sequence was analysed using online proteomics tools at the ExPASy server of the Swiss Institute of Bioinformatics (http:// www.expasy.org/tools/). Similarity searches were performed using tBLASTn and BLASTP algorithms at the National Center for Biotechnology Information (NCBI) server (http://www.ncbi.nlm. nih.gov/blast/Blast.cgi), and the FASTA3 and TFASTX algorithm at The European Bioinformatics Institute (EBI) server (http://www.ebi. ac.uk/Tools/similarity.html).

Expression and purification of recombinant cnispin (rCnp). $\mathrm{NdeI}$ and BamHI restriction sites were introduced into the $5^{\prime}$ and $3^{\prime}$ ends of the cnispin cDNA clone (GenBank accession no. GQ141891) in PCR using Pfu DNA Polymerase (Promega). After NdeI/BamHI (New England Biolabs) digestion of both insert and vectors the insert was subcloned into pET3a and pET11a vectors (Novagen) to generate recombinant proteins without tags. Both expression vectors were transformed into BL21(DE3) (Invitrogen) and BL21(DE3) pLysS (Novagen) strains of E. coli. Transformed E. coli strains were grown in Luria-Bertani (LB) medium supplemented with appropriate antibiotics at $37{ }^{\circ} \mathrm{C}$. When $\mathrm{OD}_{550}$ reached $0.5-1$, IPTG was added to a final concentration of $0.4 \mathrm{mM}$ for strains transformed with the pET3a construct and $1 \mathrm{mM}$ for strains transformed with the pET11a construct. Protein expression was monitored hourly to determine the time of maximum expression by analysing a sample of cells.

For expression of rCnp, the pET11a construct transformed into E. coli BL21(DE3) was used. Five hours after induction of expression, cells were harvested by centrifugation, resuspended in buffer $\mathrm{C}(50 \mathrm{mM}$ Tris/HCl, $2 \mathrm{mM}$ EDTA, $0.1 \%$, v/v, Triton X-100, $\mathrm{pH} 8$ ), frozen and thawed three times, then sonicated at $4{ }^{\circ} \mathrm{C}$. The insoluble fraction was separated by centrifugation ( $4000 \mathrm{~g}, 15 \mathrm{~min}$ ), resuspended in buffer C and subsequently in the same buffer containing first $2 \mathrm{M}$ urea and then $8 \mathrm{M}$ urea. The sample was applied to a Sepharose S-200 column $(4 \times 110 \mathrm{~cm})$ equilibrated with buffer $\mathrm{A}$ and inhibitory active fractions were pooled.

Circular dichroism (CD). CD spectra of purified recombinant cnispin $\left(0.45 \mathrm{mg} \mathrm{ml}^{-1}\right.$ in $0.01 \mathrm{M}$ phosphate buffer, $\mathrm{pH}$ 7) were acquired using an Aviv model 60 spectropolarimeter as described previously (Kidrič et al., 2002). Equilibrium thermal unfolding transitions were obtained using the Aviv temperature scanning facility at $232 \mathrm{~nm}$ with $0.045 \mathrm{mg} \mathrm{rCnp} \mathrm{ml} \mathrm{m}^{-1}$ in a $1 \mathrm{~cm}$ cell. Bandwidth was $2 \mathrm{~nm}$, equilibration time $5 \mathrm{~min}$, and data acquisition time $10 \mathrm{~s}$, with measurements at $2{ }^{\circ} \mathrm{C}$ intervals.

Thermal and pH stability. To test thermal stability, cnispin $\left(0.13 \mathrm{mg} \mathrm{ml}^{-1}\right)$ was incubated at $100{ }^{\circ} \mathrm{C}, 70{ }^{\circ} \mathrm{C}, 60{ }^{\circ} \mathrm{C}$ and $40{ }^{\circ} \mathrm{C}$ for $10 \mathrm{~min}$ and then at room temperature for $30 \mathrm{~min}$. To test $\mathrm{pH}$ stability, cnispin $\left(0.13 \mathrm{mg} \mathrm{ml}^{-1}\right)$ was incubated in $0.2 \mathrm{M}$ Tris/HCl ( $\mathrm{pH} 11)$, in $0.2 \mathrm{M}$ Tris/ $\mathrm{HCl}(\mathrm{pH} 7)$ or in $0.2 \mathrm{M}$ citric acid ( $\mathrm{pH} 3)$ for $20 \mathrm{~min}$ and then neutralized. Residual inhibitory activity was measured against trypsin using BAPNA as substrate.

Inhibition assay. Inhibitory activities of samples during the isolation procedure were measured against trypsin $(0.1 \mu \mathrm{M})$ in buffer $\mathrm{B}$ (0.05 M Tris/HCl, $0.02 \mathrm{M} \mathrm{CaCl}_{2}, \mathrm{pH} \mathrm{8)}$. After $10 \mathrm{~min}$ of preincubation at room temperature, $10 \mu \mathrm{l}$ of $0.1 \mathrm{M}$ substrate BAPNA 
was added and the mixture incubated for $20 \mathrm{~min}$ at $37^{\circ} \mathrm{C}$. The reaction was stopped with $0.2 \mathrm{M} \mathrm{HCl}$ and $A_{405}$ measured.

Active-site titration. The molar concentration of active trypsin was determined by titration with $p$-nitrophenyl- $p^{\prime}$-guanidinobenzoate (Chase \& Shaw, 1969). Active concentration of rCnp was determined by titration of previously active-site-titrated trypsin, using BAPNA as substrate.

Determination of inhibition constants. Inhibition kinetics of trypsin were determined under pseudo-first-order conditions in continuous assays, as described for papain inhibition by clitocypin (Brzin et al., 2000), using substrate Z-Phe-Arg-MCA and buffer B. Data were analysed by nonlinear regression analysis according to Morrison (1982), and $k_{\mathrm{d}}$ and $k_{\mathrm{a}}$ values were obtained using a $K_{\mathrm{m}}$ of $59 \mu \mathrm{M}$ for trypsin.

The inhibition kinetics for the inhibition of chymotrypsin, subtilisin, thrombin, elastase and kallikrein were determined according to Henderson (1972), as described for cathepsin B inhibition by clitocypin (Brzin et al., 2000), with the following modifications. Different amounts of the inhibitor $(0.05-8 \mu \mathrm{M})$ were incubated with each of the enzymes for $10 \mathrm{~min}$ in buffer $\mathrm{B}$ in microtitre plates. Kallikrein was assayed in $0.05 \mathrm{M}$ Tris/ $\mathrm{HCl}, 0.05 \mathrm{M} \mathrm{NaCl}, 0.01 \%(\mathrm{v} / \mathrm{v})$ Tween buffer, $\mathrm{pH} 7.8$, subtilisin in $0.1 \mathrm{M}$ phosphate buffer, $\mathrm{pH} 8.8$, and thrombin in $0.25 \mathrm{M}$ phosphate buffer, $\mathrm{pH}$ 6.5. Reactions were initiated by adding substrate to a final concentration of $30 \mu \mathrm{M}$. SucAla-Ala-Pro-Phe-MCA was used for chymotrypsin and subtilisin, $\mathrm{H}$ Pro-Phe-Arg-MCA for kallikrein, Boc-Val-Pro-Arg-MCA for thrombin and Suc-Ala-Ala-Ala-MCA for elastase. After $10 \mathrm{~min}$ of incubation, the reaction was stopped with $0.2 \mathrm{M} \mathrm{HCl}$. The released MCA was measured using a Safire microplate reader (Tecan).

Inhibitory activity against other classes of proteases. Inhibition of cysteine protease papain activity was assayed using substrate benzoyl-Arg-2-naphthylamide as described previously (Brzin et al., 2000). Inhibition of porcine pepsin, an aspartic protease, was assayed in $0.1 \mathrm{M}$ acetate buffer, $\mathrm{pH} 3.5$, with the fluorogenic substrate FTChaemoglobin, as described for FTC-casein (Twining, 1984).

Immunoblot analysis. The expression of cnispin in different parts of the basidiocarp and in cultured mycelium was analysed using aqueous extracts containing the whole soluble protein fraction (Sabotic et al., 2006). Polyclonal antibodies produced against purified rCnp in rabbits (Biogenes) were used at 1:7000 dilution following the immunoblot procedure described previously (Sabotič et al., 2006), modified by using nitrocellulose membrane (Porablot NCP, Macherey-Nagel) and chemiluminescence detection with LumiLight $^{\text {PLUS }}$ (Roche).

\section{RESULTS}

\section{Isolation and purification of C. nebularis serine protease inhibitors (CnSPIs)}

CnSPIs were obtained from a crude protein extract of $C$. nebularis basidiocarps by trypsin-affinity chromatography. An additional purification step using size-exclusion chromatography yielded a single, symmetrical protein peak with trypsin-inhibitory activity. SDS-PAGE analysis of the inhibitory fraction revealed two protein bands with estimated molecular masses of $18 \mathrm{kDa}$ and $16 \mathrm{kDa}$ (see Fig. 4a, lane 1). Ion-exchange, hydrophobic interaction and HPLC chromatographies were not successful in separating them. N-terminal sequences were, however, determined from both bands. The upper band, with apparent molecular mass $18 \mathrm{kDa}$, yielded one main sequence, LPSDQYYIRNGRSFAGRALHEDHSL, and was named CnSPI1 or cnispin. In the lower band with apparent molecular mass $16 \mathrm{kDa}$, a 16 amino acid N-terminal sequence SRPGLYFIRNGESPAG was obtained (CnSPI2 in Fig. 2) that shows $50 \%$ sequence identity to the corresponding 16 amino acid sequence of CnSPI1. In addition, a weaker sequence NLNNIVVIERTQAVH was detected in the lower band (CnSPI3 in Fig. 2). It shows no significant similarity to any of the CnSPI variants, but $33 \%$ sequence identity to the sequence of the serine protease inhibitor from Lentinus edodes (Odani et al., 1999). The internal sequence of CnSPI1 was determined by in-gel digestion with $S$. aureus V8 protease. Three peptides with apparent molecular masses of $4 \mathrm{kDa}, 7 \mathrm{kDa}$ and $10 \mathrm{kDa}$ were obtained and their $\mathrm{N}$-terminal sequences determined as LPSDQYY, DHSLNPKPVIXPTD and ALPNHGYILRARGAPTAE (underlined in Fig. 1). These sequences were used for degenerate primer design to obtain the nucleotide sequence coding for cnispin (Cnp).

\section{Characterization of CnSPIs}

ESI-mass spectrometry analysis of the HPLC-purified CnSPIs revealed two peaks with molecular masses of $16.380 \mathrm{kDa}$ and $14.321 \mathrm{kDa}$, probably corresponding to the upper and lower bands resolved in SDS-PAGE analysis. The isoelectric points of the CnSPIs were 4.8 and 5.2. The native CnSPIs were stable across a wide $\mathrm{pH}$ range, retaining their inhibitory activity after incubation at $\mathrm{pH} 2$ or $\mathrm{pH} 11$. Their inhibitory activity was lost after heating at $100{ }^{\circ} \mathrm{C}$ for $10 \mathrm{~min}$. CnSPIs proved to be very strong inhibitors of trypsin, while inhibition of chymotrypsin was about 40 times weaker. Elastase and thrombin were not inhibited by CnSPIs.

\section{Inhibition of endogenous serine proteases}

Size-exclusion chromatography of crude protein extract from basidiocarps of $C$. nebularis resolved the proteolytic activity measured against Z-Phe-Arg-MCA at $\mathrm{pH} 9$ into four peaks (Supplementary Fig. S1) with estimated molecular masses ranging from approximately $50 \mathrm{kDa}$ to $10 \mathrm{kDa}$. These proteases are of the serine catalytic type, proteolytic activity in all four peaks being completely inhibited by Pefabloc, while E-64 had no effect. Chymostatin completely inhibited activity in peaks 1 and 3 and only partially in peaks 2 and 4. CnSPIs inhibited activity of all four partially separated proteases, with $66 \%$ inhibition in peak $1,75 \%$ in peak $2,74 \%$ in peak 3 and $55 \%$ in peak 4 .

\section{Insecticidal activity against $\boldsymbol{D}$. melanogaster}

CnSPIs and other protease inhibitors were incorporated into rearing medium and their insecticidal activity against D. melanogaster was assessed. The relative mortality of larvae reared on medium containing CnSPIs was 
Trypsin inhibitors from a basidiomycete mushroom

$\begin{array}{ll}\text { Cnp-gDNA } & -190 \text { ACTATAGGGCACGCGTGGTCGACGGCCCGGGCTGGTATCGATTTGAAGTTTGAAGGATATTCCATATTTAATGAATATGT -111 } \\ \text { Cnp-gDNA } & -110 \text { CCGGGCCGCGCTGCCCCACCCAGAGGTATAAAGTCTGACAACTTCAGCCCTTTACCACTCATCATTGGAGTCCAACACCI -31 }\end{array}$

Cnp-gDNA -30 CAGACCAAGACTAATACCATTCTTATCTTGATGTCTTTTAAATTGCCTAGTGACCAATACTACATTCGGAACGGGAGGT 49 Cnp-CDNA - 30 CAGACCAAGACTAATACCATTCTTATCTTGATGTCTTTTAAATTGCCTAGTGACCAATACTACATTCGGAACGGGAGGTC 49 $\begin{array}{lllllllllllllllllllllll}\text { Deduced AA } & 1 & \text { M } & S & F & K & \text { L } & \text { P } & S & D & Q & Y & Y & I & R & N & G & S & 17\end{array}$

Cnp-gDNA 50 CTTTGCGGGTCGTGCACTCCACGAGGACCATAGCCTGAACCCGAAGCCAGTTATCTGCCCTACCGACGACACCAATGACC 129 Cnp-cDNA 50 CTTTGCGGGTCGTGCACTCCACGAGGACCATAGCCTGAACCCGAAGCCAGTTATCTGCCCTACCGACGACACCAATGACC 129

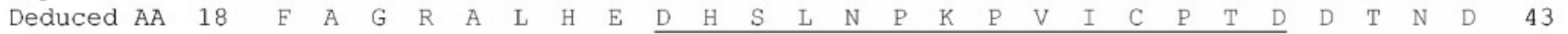

Cnp-gDNA 130 TAGCGAGTGCCCGGTGTTTTGTTGGTGGCACAATCTAACCTAACCCGTATTACGCTTCTACAGTGGATAGTTGAAGCGCT 209

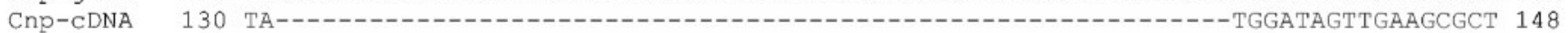

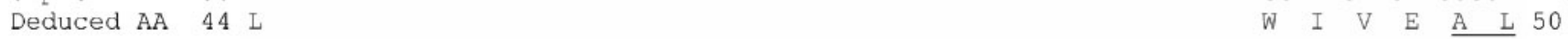

Cnp-gDNA 210 CCCAAACGGCCACTACATCCTCAGGGCCCGTGGGGCCCCCACCGCTGAACATGATGGCTATGTTTATGCTTTCTTACAAG 289 Cnp-cDNA 149 CCCAAACGGCCACTACATCCTCAGGGCCCGTGGGGCCCCCACCGCTGAACATGATGGCTATGTTTATGCTTTCTTACAAG 228

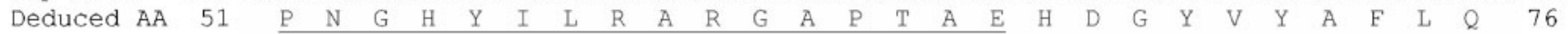

Cnp-gDNA 290 AgCGTGAGGAGAAGAAGGAATGGATTATTACCCTCCGCCCAGACCAGGACCATCAATATACGTAAGTCTTCGCATTTCCC 369

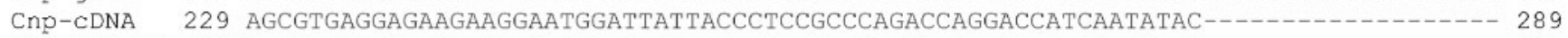

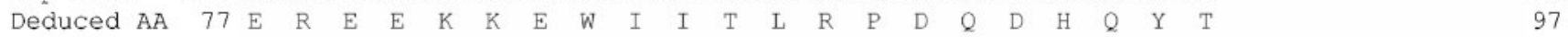

Cnp-gDNA 370 AATGTAATGCTGCTCACAgtTCTTGCAGCATCCAAGAAGCGACGGGGAAAGGCTGGATAGCGGAGACAGGCGATGAGGGC 449 Cnp-cDNA 290 -------------------CATCCAAGAAGCGACGGGGAAAGGCTGGATAGCGGAGACAGGCGATGAGGGC 341 Deduced AA 98 I $Q$ \& $E$ A

Cnp-gDNA 450 CATCAGCGGGTAAGTCTATTGGAAGCGCTCTACAGGATTCTCACCTGAATATACCATCTCTAGATTGCTGTGGGTCCCCT 529

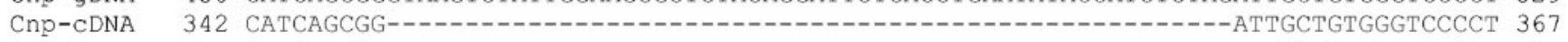
$\begin{array}{lllllllllllll}\text { Deduced AA } 115 & \text { H } & \text { Q } & \text { R } & \text { I } & \text { A } & \text { V G P L } 123\end{array}$

Cnp-gDNA 530 CCCGACCACCAAGAGTGACCCACCGCAGTTTTTGGGAAGCGCACTCTGGACCATCCAACCGCTTGTTGATTGAGTGAAAA 609 Cnp-CDNA 368 CCCGACCACCAAGAGTGACCCACCGCAGTTTTTGGGAAGCGCACTCTGGACCATCCAACCGCTTGTTGATTGAGTGAAAA 447

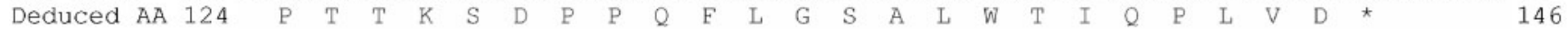

Cnp-gDNA 610 TTGTAGGAGGGGGACGAGAAAgGCCAAGAATCAAATTGACTAAATTCGTTGTAAATATGCCATTACCAATGCTAGGATTA 689 Cnp-cDNA 448 TTGTAGGAGGGGGACGAGAAAGGCCAAGAATCAAATTGACTAAATTCGTTGTAAATATGCCATTACCAATGCTAGGATTA 527

Cnp-gDNA 690 TGTGTTTCGCCCGTCAACCGAGATTTATATTTACACTAAAACCTCAAGAGTAATCCCTGAGTACTGTTGTTTTGTGCGGT 769

Cnp-CDNA 528 TGTGTTTCGCCCGTCAAAAAAAAAAAAAAAAAAAAAAAA

567

Cnp-gDNA 770 GTTGGGTATTCCCAATATGTCGAATTAGCAGCCTACGCGGCGGCTGTATGCAGAACAAGTCCGTTACGCACATAACGCTG 849

Cnp-gDNA 850 GACCCCTTCGAGGGTGTCACAGCACAGCGCAATAGGCTCAATGTAAAGTCCAGGATGCCGAAAAATCAGCCGAGAGCCCT 929

Cnp-gDNA 930 TTTAAATCTTGCCTTGTACAAGTTGTCAAAATAAGGGTATCTAATAGAGAGGCCGTAAGTTCCCAGAGAGTTTTAAAATA 1009

Fig. 1. Full-length cnispin (Cnp) gene and cDNA sequences. The genomic sequence is marked as gDNA and introns are indicated with hyphens in the cDNA sequence. The deduced amino acid sequence is shown below the nucleotide sequences. Nucleotides and amino acids are numbered starting with the start codon (ATG). The stop codon (TGA) is indicated by an asterisk. The predicted TATA box is shaded in black and the predicted transcription initiation site in grey. Underlined amino acid residues were determined by amino acid sequencing of peptides after digestion of CnSPI1 with V8 protease. The dotted line indicates the determined N-terminal amino acid sequence. GenBank accession numbers: Cnp-gDNA, FJ478178; Cnp-cDNA, GQ141891. 

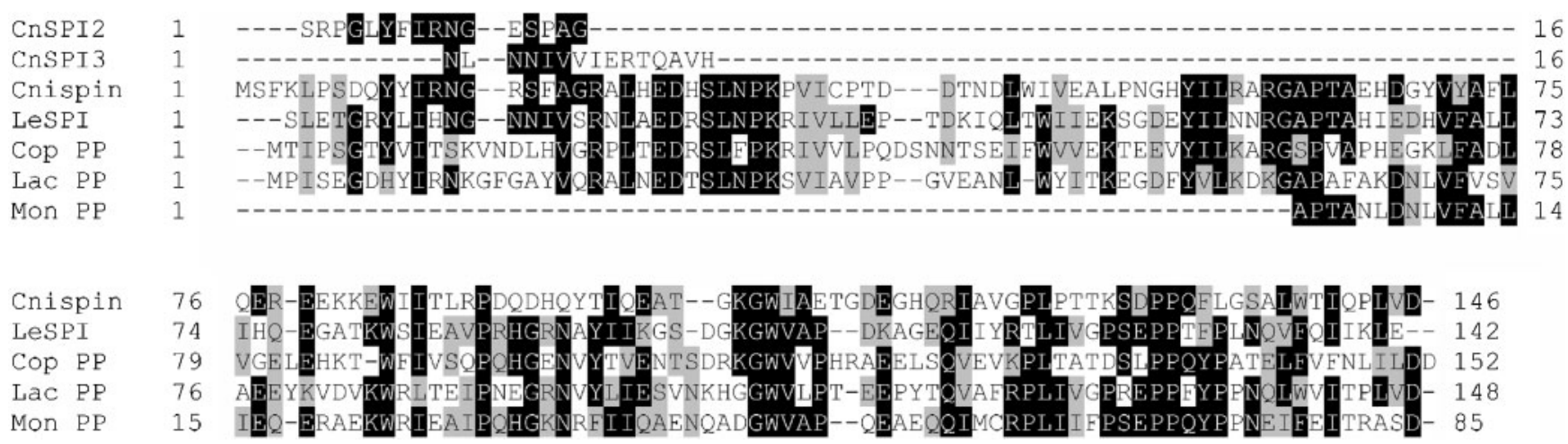

Fig. 2. Alignment of amino acid sequences of similar serine protease inhibitors from different basidiomycetes. Amino acid sequences of $\mathrm{CnSPls}$ and the cnispin deduced amino acid sequence are aligned with the amino acid sequence of the Lentinus edodes serine protease inhibitor (Odani et al., 1999) (LeSPI, Swiss-Prot P81639), and the predicted protein sequences annotated in the Coprinopsis cinerea genome (Cop PP, GenBank AACS01000321), Laccaria bicolor genome (Lac PP, GenBank DS547153) and Moniliophthora perniciosa genome (Mon PP, GenBank EEB87837). Identical amino acid residues are shaded in black and similar amino acid residues in grey.

$0.59 \pm 0.22$. While potato protease inhibitor 2 (PI2) showed a very strong insecticidal activity, with no larvae surviving, the activity of CnSPIs was similar to that of another potato serine protease inhibitor, PSPI, that produced a relative mortality of $0.46 \pm 0.08$. In contrast, the serine protease inhibitor from pumpkin (CMTI) had almost no effect, with relative mortality $0.03 \pm 0.00$, which was similar to that of the cysteine protease inhibitor from C. nebularis, clitocypin, with relative mortality $0.07 \pm 0.02$.

\section{Analysis of the cnispin gene and cDNA sequences}

The complete sequence of the cnispin gene, with its promoter and terminator sequences, was obtained. Comparison of the full-length gene with the cDNA sequence revealed that the $603 \mathrm{bp}$ coding region is composed of four exons $(132,158,61,90 \mathrm{bp})$ and three short introns $(61,47,54 \mathrm{bp})$ (Fig. 1). The intron-exon boundaries in the second and third intron follow the GTAG consensus splice sites, which are GURAGU for the 5' splice site and WNYAG for the $3^{\prime}$ splice site in filamentous fungi (Kupfer et al., 2004). The first intron, however, contains the variant GC-AG splice site pair, which is present in $1.89 \%$ of introns in the basidiomycete Cryptococcus neoformans and in 0.86-1.19\% of introns in the ascomycetes Neurospora crassa, Aspergillus nidulans and Saccharomyces cerevisiae (Kupfer et al., 2004). The cnispin promoter has a putative consensus TATA box sequence (TATAAA) at position -84 and a putative transcription initiation site (TCAGACC) at position -31 (Fig. 1), indicating transcriptional activity of the cnp gene. Similar positions were observed in the promoters of genes coding for a cysteine protease inhibitor, clitocypin (Sabotič et al., 2006), and a ricin B-like lectin from C. nebularis (Pohleven et al., 2009). As for these genes, no typical polyadenylation signal AAUAAA was found in the cnp 3' UTR sequence.

\section{Cnispin amino acid sequence analysis}

The coding region of cnispin cDNA is 441 bp long (stop codon included) and translates into a 146 amino acid protein. The deduced amino acid sequence of cnispin matched exactly the corresponding parts of the amino acid sequence that was determined by protein sequencing (underlined in Fig. 1). Sequence similarity searches in databases were performed and a significant sequence similarity was found with a serine protease inhibitor from Lentinus edodes characterized at the protein level and a few hypothetical proteins annotated in genomes of basidiomycetes (Fig. 2). Cnispin exhibited the highest sequence identity $(37 \%)$ with $L$. edodes serine proteinase inhibitor (here termed LeSPI) (Odani et al., 1999), the only member of the I66 family of protease inhibitors. Furthermore, it exhibited $34 \%$ sequence identity with three predicted proteins in the Coprinopsis cinerea Okayama genome and $33 \%$ sequence identity with three predicted proteins in the Laccaria bicolor S238N-H82 genome (Martin et al., 2008). High sequence similarity was also found for limited regions of one other predicted protein in the $C$. cinerea genome and another four from the genome of L. bicolor. Similarly, sequence identity (35\%) was also found with the Cterminal half of a protein with a partial sequence annotated in the Moniliophthora perniciosa FA553 genome (Mondego et al., 2008). In contrast to LeSPI and other putative serine protease inhibitors that do not have cysteines, cnispin contains a single cysteine in its amino acid sequence (C36).

\section{Expression and purification of active recombinant cnispin}

Purification of individual CnSPIs from basidiocarps could not be achieved due to their very similar biochemical properties; therefore, cnispin was heterologously expressed in $E$. coli for further characterization. The cDNA clone 
(GenBank accession no. GQ141891; Fig. 1) was subcloned into expression vectors pET3a and pET11a and they were both transformed into E. coli BL21(DE3) and BL21(DE3) pLysS to determine the optimal expression system. Although cnispin was expressed in all the tested combinations, maximum expression of recombinant cnispin (rCnp) was achieved $5 \mathrm{~h}$ after induction using E. coli BL21(DE3) with expression vector pET1la::rCnp (Fig. 3). The inhibitor was expressed mainly as insoluble inclusion bodies (Fig. 3). Progressive solubilization of the pellet in $2 \mathrm{M}$ and $8 \mathrm{M}$ urea, followed by a one-step purification using size-exclusion chromatography, yielded a purified protein at a yield of $70 \mathrm{mg} \mathrm{rCnp}$ per litre of bacterial culture (Fig. 3). The purified rCnp exhibited a single $18 \mathrm{kDa}$ band on SDS-PAGE under reducing conditions (Fig. 4a, lane 2). Under non-reducing conditions, however, it showed an additional band with an apparent molecular mass of $32 \mathrm{kDa}$, corresponding to a dimer, probably formed between the single cysteine residues.

\section{Characterization of recombinant cnispin}

Comparison of the molecular masses of rCnp and CnSPIs isolated from $C$. nebularis basidiocarps showed that $\mathrm{rCnp}$ indeed corresponds to the higher-molecular-mass protein CnSPI1 (Fig. 4a). Furthermore, polyclonal antibodies raised against $\mathrm{rCnp}$ recognized the upper CnSPI1 band

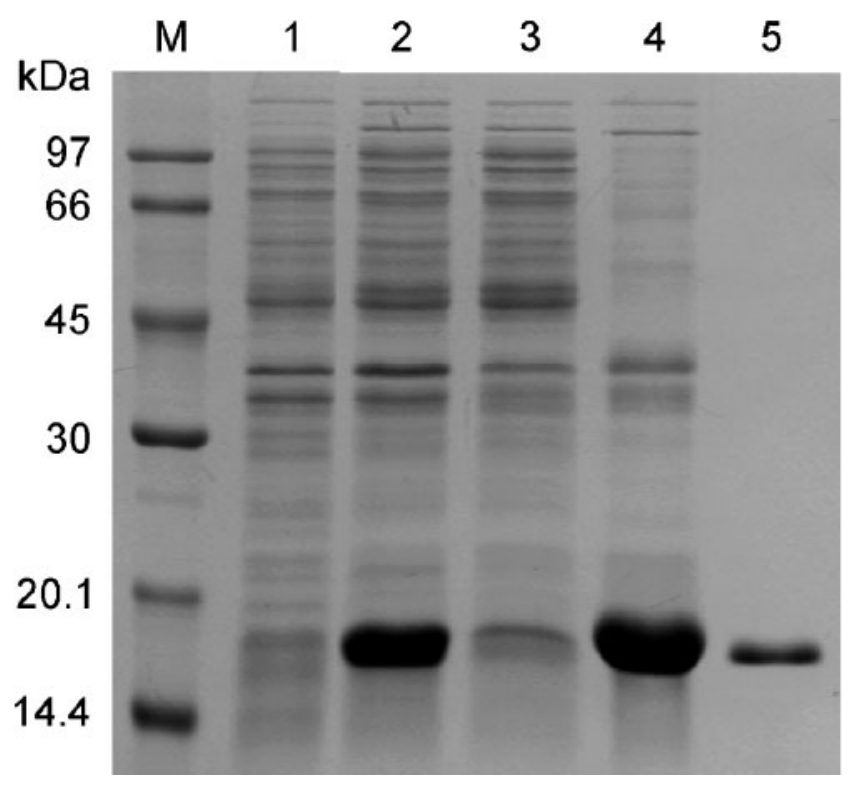

Fig. 3. Expression and purification of recombinant cnispin. Recombinant cnispin was analysed by SDS-PAGE on $12 \%$ $(w / v)$ PAA gel under reducing conditions and stained with Coomassie blue. Lanes 1 and 2, crude cell extracts of E. coli BL21(DE3)/pET11a: : rCnp before (lane 1) and $5 \mathrm{~h}$ after (lane 2) induction of expression. Lane 3 , soluble fraction of cell lysate; lane 4 , insoluble fraction of cell lysate dissolved in $8 \mathrm{M}$ urea; lane 5 , purified recombinant cnispin. $\mathrm{M}$, protein molecular mass markers. (a)

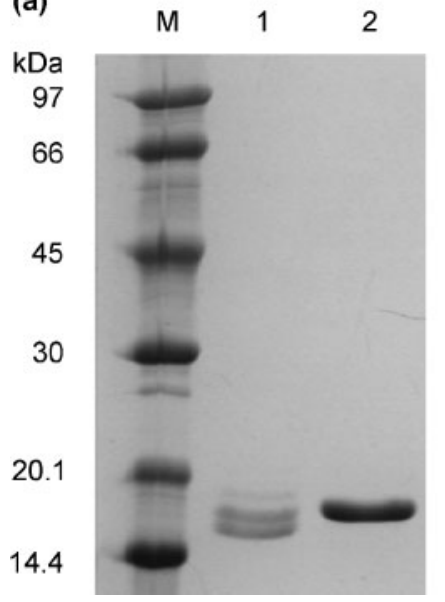

(b)

kDa

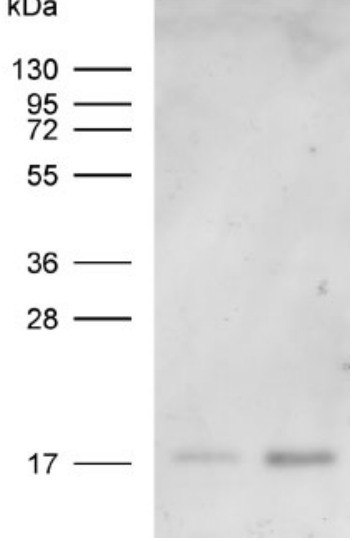

Fig. 4. Comparison of CnSPls and cnispin by SDS-PAGE (a) and immunoblot (b) analyses. (a) PAA gel and Coomassie staining was used for SDS-PAGE analysis of CnSPls and rCnp. (b) Proteins separated by SDS-PAGE under reducing conditions were transferred to nitrocellulose membrane and probed with polyclonal anti-rCnp antibodies. Immunoreactive bands were visualized by probing the blot with horseradish-peroxidase-conjugated goat anti-rabbit IgG secondary antibodies, followed by chemiluminescence detection. Lane 1, CnSPls purified from basidiocarps by trypsin affinity and size-exclusion chromatographies; lane 2, purified recombinant cnispin. $\mathrm{M}$, protein molecular mass markers.

specifically (Fig. $4 \mathrm{~b}$ ). The identity of the protein was confirmed by $\mathrm{N}$-terminal sequencing (SFKLP). The calculated molecular mass of $16.407 \mathrm{kDa}$ for rCnp lacking the initial methionine was confirmed exactly by ESI-mass spectrometry. IEF analysis confirmed the theoretical isoelectric point of 5.3.

The CD spectrum for rCnp in the far UV (Supplementary Fig. S2) showed marked similarity to that of clitocypin (Clt) (Kidrič et al., 2002; Sabotič et al., 2007a). The peak at $232 \mathrm{~nm}$, which is a marker for tryptophan environment, is less marked than for Clt, but is still sensitive to unfolding (Supplementary Fig. S3). The only marked difference is that no peak is observed at $189 \mathrm{~nm}$. While the contribution of tryptophan prevents an analysis of secondary structure in terms of $\beta$ structure, there is clearly little or no $\alpha$-helical structure.

The thermal stability of rCnp was examined by following its unfolding by $\mathrm{CD}$. The temperature unfolding transition (Supplementary Fig. S3) shows a temperature midpoint of $56{ }^{\circ} \mathrm{C}$, considerably less than that for the cysteine protease inhibitor clitocypin, at $67{ }^{\circ} \mathrm{C}$ (Kidrič et al., 2002; Sabotič et al., 2007a). The limited thermal stability of rCnp was also indicated by measurements of inhibitory activity. It exhibited full inhibitory activity after 10 min incubation at $40{ }^{\circ} \mathrm{C}$, while at higher temperatures inhibition was reduced. No activity was retained after heating at $100{ }^{\circ} \mathrm{C}$ for $10 \mathrm{~min}$. On the other hand, $\mathrm{rCnp}$ was stable across a 
wide $\mathrm{pH}$ range, retaining its inhibitory activity after incubation at $\mathrm{pH} 3$ and $\mathrm{pH} 11$.

\section{Kinetics of inhibition}

Titration of trypsin with cnispin showed that complete inhibition was achieved at 1:1 molar stoichiometry. The pseudo-first-order rate constant, $k$, for binding of rCnp to trypsin increased linearly with inhibitor concentration. Kinetic constants of the inhibition of different proteases by rCnp are presented in Table 1. rCnp was most effective in inhibiting trypsin, with a $K_{\mathrm{i}}$ value of $3.1 \mathrm{nM}$, showing it to be a fast-acting $\left(k_{\mathrm{a}} 3.1 \times 10^{5} \mathrm{M}^{-1} \mathrm{~s}^{-1}\right)$ and tight-binding $\left(k_{\mathrm{d}} 9.5 \times 10^{-4} \mathrm{~s}^{-1}\right)$ inhibitor of this enzyme. The $K_{\mathrm{i}}$ value for the inhibition of chymotrypsin was in the micromolar range, while those for subtilisin and porcine kallikrein were significantly higher. rCnp showed no inhibitory activity against the other serine proteases porcine elastase and bovine thrombin, nor against the cysteine protease papain or the aspartic protease pepsin. It was also established that rCnp does not inhibit the human serine proteases $\beta$ tryptase, coagulation factors Xa and IXa, plasmin, urokinase, polymorphonuclear leukocyte elastase, thrombin or kallikrein 5 (not shown).

\section{Expression of cnispin in the basidiocarp and in cultured mycelium}

Detection of cnispin in different parts of the basidiocarp using polyclonal anti-rCnp antibodies revealed its presence in all parts of the basidiocarp. Analysis of the distribution showed less cnispin in the stipe and more in other parts of the basidiocarp, particularly in the cap and lamellae (Fig. 5). Cnispin was also expressed in cultured mycelium (Fig. 5), but was not secreted into the medium (not shown).

Table 1. Kinetic constants for the interaction of cnispin with different proteases

Kinetic and equilibrium constants for the inhibition of trypsin were determined under pseudo-first-order conditions in a continuous kinetic assay according to Morrison (1982). Equilibrium constants for the inhibition of chymotrypsin, subtilisin and kallikrein were determined according to Henderson (1972). Experiments were performed at $25{ }^{\circ} \mathrm{C}$. Standard deviation is given where appropriate; ND, not determined; NI, no inhibition.

\begin{tabular}{|c|c|c|c|}
\hline Enzyme & $K_{\mathrm{i}}(\mathrm{nM})$ & $10^{-5} \times k_{\mathrm{a}}\left(\mathrm{M}^{-1} \mathrm{~s}^{-1}\right)$ & $10^{4} \times k_{\mathrm{d}}\left(\mathrm{s}^{-1}\right)$ \\
\hline Trypsin & $3.10 \pm 0.66$ & $3.10 \pm 0.55$ & $9.50 \pm 0.47$ \\
\hline Chymotrypsin & $120 \pm 20$ & ND & $\mathrm{ND}$ \\
\hline Subtilisin & $>1000$ & ND & ND \\
\hline Kallikrein & $>1000$ & ND & ND \\
\hline Elastase & NI & & \\
\hline Thrombin & NI & & \\
\hline Papain & NI & & \\
\hline Pepsin & NI & & \\
\hline
\end{tabular}

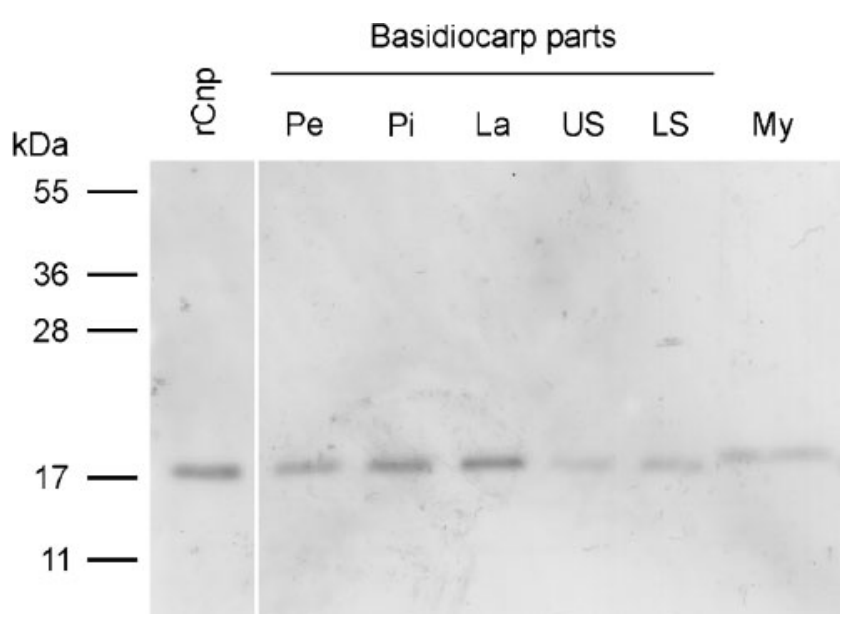

Fig. 5. Expression of cnispin in different parts of the basidiocarp and in cultured mycelium. Immunoblot analysis of cnispin expression in aqueous extracts was performed using polyclonal anti-rCnp antibodies. rCnp, recombinant cnispin; $\mathrm{Pe}$, pellis; $\mathrm{Pi}$, pileus; La, lamellae; US, upper stipe; LS, lower stipe; My, mycelium.

Similarity searches of the expressed sequence tags (EST) databases revealed a number of ESTs showing similarity to the cnispin amino acid sequence. They further support the expression pattern of cnispin-like serine protease inhibitors, as they were determined from both vegetative mycelia and fruiting bodies of the basidiomycete mushrooms Laccaria bicolor, Coprinopsis cinerea and Lentinus edodes.

\section{DISCUSSION}

Serine protease inhibitors, CnSPIs, were purified from basidiocarps of $C$. nebularis using trypsin affinity chromatography. On SDS-PAGE analysis (Fig. 4a) they were present as two bands which revealed three $\mathrm{N}$-terminal sequences, all showing significant sequence similarity to the serine protease inhibitor from Lentinus edodes (LeSPI) (Odani et al., 1999), until now the sole member of the I66 family of protease inhibitors in the MEROPS classification. The similar biochemical properties of the CnSPIs and the strong sequence similarity of their $\mathrm{N}$-terminal sequences suggested that they all belong to the I66 protease inhibitor family. Since attempts to isolate the individual CnSPIs were not successful, CnSPI1, which showed the highest sequence similarity with LeSPI, was further characterized and named cnispin (Cnp). Based on the partial protein sequences obtained for cnispin, its coding gene and corresponding cDNA were amplified and sequenced, together with the promoter and $5^{\prime}$ UTR and $3^{\prime}$ UTR sequences (Fig. 1). Comparison of the full-length LeSPI protein sequence (142 amino acids) with the deduced amino acid sequence of cnispin (145 amino acids) revealed many conserved amino acid residues distributed throughout the sequence (Fig. 2), which are probably important for the inhibitory activity 
and/or structure of the proteins. The single cysteine that is present in cnispin is probably responsible for the dimer formation observed for recombinant cnispin (rCnp) on SDS-PAGE under non-reducing conditions. A dimeric form of cnispin was also detected in basidiocarps by immunoblot analysis. Dimerization could represent a regulatory mechanism, since dimeric rCnp did not inhibit trypsin.

Biochemical characterization of CnSPIs and rCnp revealed them to have similar properties. On SDS-PAGE analysis under reducing conditions rCnp corresponded to the upper band of CnSPIs, which was confirmed by the similar molecular masses determined by ESI-mass spectrometry (16.407 kDa for rCnp, and $16.380 \mathrm{kDa}$ and $14.321 \mathrm{kDa}$ for CnSPIs). The apparent molecular masses observed on SDSPAGE analysis are higher. CnSPIs and rCnp, like LeSPI (Odani et al., 1999), showed similar isoelectric points, exhibited stability over a wide $\mathrm{pH}$ range and lost their inhibitory activity after incubation at high temperatures. However, the thermal stability of CnSPIs and rCnp is much lower than that of the thermally stable proteins clitocypin (Sabotic et al., 2007a) and Clitocybe nebularis lectin (Pohleven et al., 2009), also isolated from C. nebularis.

Cnispin inhibited trypsin with high specificity, with $K_{\mathrm{i}}$ in the nanomolar range. It inhibited chymotrypsin with $K_{\mathrm{i}}$ in the micromolar range, showed an even weaker inhibition of subtilisin and kallikrein, and no inhibition of the other serine proteases tested. The inhibitory profile of cnispin (Table 1) is very similar to that of LeSPI, which is also a strong inhibitor of trypsin and a weak inhibitor of chymotrypsin, while other serine proteases are not inhibited (Odani et al., 1999). In addition, proteases of other catalytic classes are not inhibited by either cnispin or LeSPI. Both inhibitors inhibit trypsin at 1:1 molar ratio. Arginine is believed to be the primary reactive residue of LeSPI for trypsin (Odani et al., 1999). Comparison of the amino acid sequences of cnispin, LeSPI and predicted serine protease inhibitors from other basidiomycetes (Fig. 2) revealed two conserved arginine residues, R21 and R60, either of which could be the inhibitory-active residue. An exception is the Laccaria bicolor predicted protein, which has a lysine at position 60 instead of an arginine, and shows a specificity for binding to the trypsin $S_{1}$ site similar to that for arginine (Bode \& Huber, 1992).

Inhibitory activity against endogenous proteases and insecticidal activity against $D$. melanogaster larvae suggest regulatory and defensive biological functions for CnSPIs. A regulatory role in the endogenous proteolytic system of $C$. nebularis has been suggested for CnSPIs, based on specific inhibition of serine-type proteolytic activity from different basidiomycete mushrooms, including C. nebularis (Sabotič et al., 2007b). The intracellular localization of CnSPIs supports an endogenous regulatory role. This was further confirmed by considerable inhibition of all partially purified serine proteases from C. nebularis by CnSPIs. Different levels of inhibition by Pefabloc, chymostatin and
CnSPIs of the four separated peaks of proteolytic activity from the extract indicate a difference in specificity of these proteases. A role of CnSPIs in fruiting body development and/or resource recycling is thus probable.

The insecticidal effect against the model dipteran $D$. melanogaster suggests a defensive role of CnSPIs against predatory insects. Flies (Diptera) are major mushroom pests; most of them attack at early mushroom developmental stages while others, like flies of the genus Drosophila, feed on later developmental stages or mature mushrooms (Coles et al., 2002; Krivosheina, 2008). Serine proteases constitute the predominant digestive proteolytic activity of dipterans (Terra \& Ferreira, 1994). Analogously to plants, in which protease inhibitors play an important defensive role (Habib \& Fazili, 2007), serine protease inhibitors probably play a defensive role against predation by insect pests and different pathogens also in mushrooms. The insecticidal effect of CnSPIs was compared with those of three plant serine protease inhibitors: PSPI and PI2, two serine protease inhibitors from potato (Brzin et al., 1995; Pearce et al., 1982), and CMTI, a trypsin inhibitor from pumpkin (Krishnamoorthi et al., 1990). The CnSPIs showed an effect similar to that of PSPI, PI2 left no surviving larvae, whereas CMTI had no effect. A cysteine protease inhibitor, clitocypin, from C. nebularis was also used in the bioassay, since a putative digestive cysteine protease has been identified in D. melanogaster (Matsumoto et al., 1995). Clitocypin had no effect on larval survival, confirming the predominant role of serine proteases in protein digestion of Drosophila larvae. Therefore, CnSPIs could offer an effective defence, with the possibility of adaptation and selection of the most appropriate isoforms.

In conclusion, a new serine protease inhibitor, cnispin, from the basidiomycete $C$. nebularis, has been characterized at the genetic and biochemical levels. Evidence is described that indicates its dual biological function. The similarity in primary sequence and biochemical properties to LeSPI indicates that cnispin belongs to the I66 family of protease inhibitors in the MEROPS classification. Analysis of the inhibition profile showed high specificity towards trypsinlike serine proteases. This strong and specific inhibition of trypsin, with many other serine proteases being unaffected, suggests that cnispin could prove an effective and useful tool in studying trypsin-dependent cellular processes in humans, such as cancer and inflammation of the cardiovascular, respiratory, musculoskeletal, gastrointestinal and nervous systems (Ramachandran \& Hollenberg, 2008).

\section{ACKNOWLEDGEMENTS}

The work was supported by the Slovenian Research Agency [grant no. P4-0127 (J.K.) and a Young Researchers fellowship]. We thank Professor Dr Christian P. Sommerhoff (University of Münich, Germany), Professor Dr Johann Brandstetter and Dr Thomas Zögg (University of Salzburg, Austria) for inhibitory profiling of human 
proteases. We thank Professor Dr Andrej Čokl, Dr Špela Schrader (National Institute of Biology, Ljubljana, Slovenia) and Lara Kandić for insecticidal tests. We are grateful to Dr Bogdan Kralj (Jožef Stefan Institute, Ljubljana, Slovenia) for performing ESI mass spectrometry measurements.

\section{REFERENCES}

Blumberg, S., Schechter, I. \& Berger, A. (1970). The purification of papain by affinity chromatography. Eur J Biochem 15, 97-102.

Bode, W. \& Huber, R. (1992). Natural protein proteinase inhibitors and their interaction with proteinases. Eur J Biochem 204, 433-451.

Brzin, J., Meško, P. \& Kregar, I. (1995). Potato tubers protein inhibitors of cysteine, serine and aspartic proteinases. Acta Pharm 45, 181-186.

Brzin, J., Rogelj, B., Popovič, T., Štrukelj, B. \& Ritonja, A. (2000). Clitocypin, a new type of cysteine proteinase inhibitor from fruit bodies of mushroom Clitocybe nebularis. J Biol Chem 275, 2010420109.

Burton, K. S., Partis, M. D., Wood, D. A. \& Thurston, C. F. (1997). Accumulation of serine proteinase in senescent sporophores of the cultivated mushroom, Agaricus bisporus. Mycol Res 101, 146-152.

Chase, T., Jr \& Shaw, E. (1969). Comparison of the esterase activities of trypsin, plasmin, and thrombin on guanidinobenzoate esters. Titration of the enzymes. Biochemistry 8, 2212-2224.

Coles, P. S., Barber, W., Beyer, D. M., Fleischer, S. J., Keil, C., Rinker, D. L., Romaine, C. P., Whitney, S. P. \& Wuest, P. (2002). Mushroom Integrated Pest Management. PA, USA: Pennsylvania State University.

Dohmae, N., Takio, K., Tsumuraya, Y. \& Hashimoto, Y. (1995). The complete amino acid sequences of two serine proteinase inhibitors from the fruiting bodies of a basidiomycete, Pleurotus ostreatus. Arch Biochem Biophys 316, 498-506.

Espin, J. C., van Leeuwen, J. \& Wichers, H. J. (1999). Kinetic study of the activation process of a latent mushroom (Agaricus bisporus) tyrosinase by serine proteases. J Agric Food Chem 47, 3509-3517.

Faraco, V., Palmieri, G., Festa, G., Monti, M., Sannia, G. \& Giardina, P. (2005). A new subfamily of fungal subtilases: structural and functional analysis of a Pleurotus ostreatus member. Microbiology 151, 457-466.

Gzogian, L. A., Proskuriakov, M. T., levleva, E. V. \& Valueva, T. A. (2005). Trypsin-like proteinases and trypsin inhibitors in fruiting bodies of higher fungi. Prikl Biokhim Mikrobiol 41, 612-615 (in Russian).

Habib, H. \& Fazili, K. M. (2007). Plant protease inhibitors: a defense strategy in plants. Biotechnol Mol Biol Rev 2, 068-085.

Henderson, P. J. (1972). A linear equation that describes the steadystate kinetics of enzymes and subcellular particles interacting with tightly bound inhibitors. Biochem J 127, 321-333.

Heneghan, M. N., Porta, C., Zhang, C., Burton, K. S., Challen, M. P., Bailey, A. M. \& Foster, G. D. (2009). Characterization of serine proteinase expression in Agaricus bisporus and Coprinopsis cinerea by using green fluorescent protein and the A. bisporus SPR1 promoter. Appl Environ Microbiol 75, 792-801.

Kidrič, M., Fabian, H., Brzin, J., Popovič, T. \& Pain, R. H. (2002). Folding, stability, and secondary structure of a new dimeric cysteine proteinase inhibitor. Biochem Biophys Res Commun 297, 962-967.

Krishnamoorthi, R., Gong, Y. X. \& Richardson, M. (1990). A new protein inhibitor of trypsin and activated Hageman factor from pumpkin (Cucurbita maxima) seeds. FEBS Lett 273, 163-167.

Krivosheina, N. P. (2008). Macromycete fruit bodies as a habitat for dipterans (Insecta, Diptera). Entomol Rev (Engl Transl) 88, 778-792.
Kupfer, D. M., Drabenstot, S. D., Buchanan, K. L., Lai, H., Zhu, H., Dyer, D. W., Roe, B. A. \& Murphy, J. W. (2004). Introns and splicing elements of five diverse fungi. Eukaryot Cell 3, 1088-1100.

Martin, F., Aerts, A., Ahren, D., Brun, A., Danchin, E. G., Duchaussoy, F., Gibon, J., Kohler, A., Lindquist, E. \& other authors (2008). The genome of Laccaria bicolor provides insights into mycorrhizal symbiosis. Nature 452, 88-92.

Matsumoto, I., Watanabe, H., Abe, K., Arai, S. \& Emori, Y. (1995). A putative digestive cysteine proteinase from Drosophila melanogaster is predominantly expressed in the embryonic and larval midgut. Eur $J$ Biochem 227, 582-587.

Moller, E. M., Bahnweg, G., Sandermann, H. \& Geiger, H. H. (1992). A simple and efficient protocol for isolation of high molecular weight DNA from filamentous fungi, fruit bodies, and infected plant tissues. Nucleic Acids Res 20, 6115-6116.

Mondego, J. M., Carazzolle, M. F., Costa, G. G., Formighieri, E. F., Parizzi, L. P., Rincones, J., Cotomacci, C., Carraro, D. M., Cunha, A. F. \& other authors (2008). A genome survey of Moniliophthora perniciosa gives new insights into Witches' Broom Disease of cacao. BMC Genomics 9, 548.

Morrison, J. F. (1982). The slow-binding and slow, tight-binding inhibition of enzyme-catalysed reactions. Trends Biochem Sci 7, 102105.

Muhlia-Almazan, A., Sanchez-Paz, A. \& Garcia-Carreno, F. L. (2008). Invertebrate trypsins: a review. J Comp Physiol [B] 178, 655-672.

Nyberg, P., Ylipalosaari, M., Sorsa, T. \& Salo, T. (2006). Trypsins and their role in carcinoma growth. Exp Cell Res 312, 1219-1228.

Odani, S., Tominaga, K., Kondou, S., Hori, H., Koide, T., Hara, S., Isemura, M. \& Tsunasawa, S. (1999). The inhibitory properties and primary structure of a novel serine proteinase inhibitor from the fruiting body of the basidiomycete, Lentinus edodes. Eur J Biochem 262, 915-923.

Ortiz, M. L., Calero, M., Fernandez Patron, C., Patron, C. F., Castellanos, L. \& Mendez, E. (1992). Imidazole-SDS-Zn reverse staining of proteins in gels containing or not SDS and microsequence of individual unmodified electroblotted proteins. FEBS Lett 296, 300304.

Page, M. J. \& Di Cera, E. (2008). Serine peptidases: classification, structure and function. Cell Mol Life Sci 65, 1220-1236.

Palmieri, G., Bianco, C., Cennamo, G., Giardina, P., Marino, G., Monti, M. \& Sannia, G. (2001). Purification, characterization, and functional role of a novel extracellular protease from Pleurotus ostreatus. Appl Environ Microbiol 67, 2754-2759.

Pearce, G., Sy, L., Russell, C., Ryan, C. A. \& Hass, G. M. (1982). Isolation and characterization from potato tubers of two polypeptide inhibitors of serine proteinases. Arch Biochem Biophys 213, 456462.

Pohleven, J., Obermajer, N., Sabotič, J., Anžlovar, S., Sepčić, K., Kos, J., Kralj, B., Štrukelj, B. \& Brzin, J. (2009). Purification, characterization and cloning of a ricin B-like lectin from mushroom Clitocybe nebularis with antiproliferative activity against human leukemic T cells. Biochim Biophys Acta 1790, 173-181.

Ramachandran, R. \& Hollenberg, M. D. (2008). Proteinases and signalling: pathophysiological and therapeutic implications via PARs and more. Br J Pharmacol 153, S263-S282.

Rawlings, N. D., Morton, F. R., Kok, C. Y., Kong, J. \& Barrett, A. J. (2008). MEROPS: the peptidase database. Nucleic Acids Res 36, D320D325.

Sabotič, J., Gaser, D., Rogelj, B., Gruden, K., Štrukelj, B. \& Brzin, J. (2006). Heterogeneity in the cysteine protease inhibitor clitocypin gene family. Biol Chem 387, 1559-1566. 
Sabotič, J., Galeša, K., Popovič, T., Leonardi, A. \& Brzin, J. (2007a). Comparison of natural and recombinant clitocypins, the fungal cysteine protease inhibitors. Protein Expr Purif 53, 104-111.

Sabotič, J., Trček, T., Popovič, T. \& Brzin, J. (2007b). Basidiomycetes harbour a hidden treasure of proteolytic diversity. J Biotechnol 128, 297-307.

Sabotič, J., Popovič, T., Puizdar, V. \& Brzin, J. (2009). Macrocypins, a family of cysteine protease inhibitors from the basidiomycete Macrolepiota procera. FEBS J 276, 4334-4345.

St Leger, R. J., Joshi, L. \& Roberts, D. W. (1997). Adaptation of proteases and carbohydrates of saprophytic, phytopathogenic and entomopathogenic fungi to the requirements of their ecological niches. Microbiology 143, 1983-1992.

Terra, W. R. \& Ferreira, C. (1994). Insect digestive enzymes: properties, compartmentalization and function. Comp Biochem Physiol 109, 1-62.
Twining, S. S. (1984). Fluorescein isothiocyanate-labeled casein assay for proteolytic enzymes. Anal Biochem 143, 30-34.

Vandeputte-Rutten, L. \& Gros, P. (2002). Novel proteases: common themes and surprising features. Curr Opin Struct Biol 12, 704708.

Vetter, J. (2000). Trypsin inhibitor activity of basidiomycetous mushrooms. Eur Food Res Technol 211, 346-348.

Whitcomb, D. C. \& Lowe, M. E. (2007). Human pancreatic digestive enzymes. Dig Dis Sci 52, 1-17.

Zuchowski, J. \& Grzywnowicz, K. (2006). Partial purification of proteinase $\mathrm{K}$ inhibitors from liquid-cultured mycelia of the white rot basidiomycete Trametes versicolor. Curr Microbiol 53, 259264.

Edited by: J. M. Becker 